\title{
STUDY OF SOME SUBCLASSES OF UNIVALENT FUNCTIONS AND THEIR RADIUS PROPERTIES
}

\author{
S. Ponnusamy and S. K. Sahoo
}

\begin{abstract}
An analytic function $f(z)=z+a_{2} z^{2}+\cdots$ in the unit disk $\Delta=\{z:|z|<1\}$ is said to be in $\mathscr{U}(\lambda, \mu)$ if

$$
\left|f^{\prime}(z)\left(\frac{z}{f(z)}\right)^{\mu+1}-1\right| \leq \lambda \quad(|z|<1)
$$

for some $\lambda \geq 0$ and $\mu>-1$. For $-1 \leq \alpha \leq 1$, we introduce a geometrically motivated $\mathscr{S}_{p}(\alpha)$-class defined by

$$
\mathscr{S}_{p}(\alpha)=\left\{f \in \mathscr{S}:\left|\frac{z f^{\prime}(z)}{f(z)}-1\right| \leq \operatorname{Re} \frac{z f^{\prime}(z)}{f(z)}-\alpha, z \in \Delta\right\},
$$

where $\mathscr{S}$ represents the class of all normalized univalent functions in $\Delta$. In this paper, the authors determine necessary and sufficient coefficient conditions for certain class of functions to be in $\mathscr{S}_{p}(\alpha)$. Also, radius properties are considered for $\mathscr{S}_{p}(\alpha)$-class in the class $\mathscr{S}$. In addition, we also find disks $|z|<r:=r(\lambda, \mu)$ for which $\frac{1}{r} f(r z) \in \mathscr{U}(\lambda, \mu)$ whenever $f \in \mathscr{S}$. In addition to a number of new results, we also present several new sufficient conditions for $f$ to be in the class $\mathscr{U}(\lambda, \mu)$.
\end{abstract}

\section{Introduction and preliminaries}

Denote by $\mathscr{A}$ the class of all functions $f$, normalized by $f(0)=0=$ $f^{\prime}(0)-1$, that are analytic in the unit disk $\Delta=\{z \in \mathbf{C}:|z|<1\}$, and by $\mathscr{S}$ the class of univalent functions $f \in \mathscr{A}$. Denote by $\mathscr{S}^{*}$ the subclass consisting of functions $f$ in $\mathscr{S}$ that are starlike (with respect to origin), i.e. $t w \in f(\Delta)$ whenever $t \in[0,1]$ and $w \in f(\Delta)$. Analytically, $f \in \mathscr{S}^{*}$ if and only if $\operatorname{Re}\left(z f^{\prime}(z) / f(z)\right) \geq 0$ in $\Delta$. A simple generalization of $\mathscr{S}^{*}$ is the so-called class of all starlike functions of order $\alpha, 0 \leq \alpha \leq 1$, denoted by $\mathscr{S}^{*}(\alpha)$. Indeed, $f \in \mathscr{S}^{*}(\alpha)$ if and only

2000 Mathematics Subject Classification. $30 \mathrm{C} 45$.

Key words and phrases. Coefficient inequality, analytic, univalent and starlike functions.

The work was initiated during the visit of the first author to the University of Turku, Finland. The visit was supported by Commission on Development and Exchanges of the International Mathematical Union and this author thanks CDE for its support.

Received November 30, 2005; revised May 8, 2006. 
if $\operatorname{Re}\left(z f^{\prime}(z) / f(z)\right) \geq \alpha$ in $\Delta$. We set $\mathscr{S}^{*}(0)=\mathscr{S}^{*}$. A function $f \in \mathscr{A}$ is said to be in $\mathscr{U}(\lambda, \mu)$ if

$$
\left|f^{\prime}(z)\left(\frac{z}{f(z)}\right)^{\mu+1}-1\right| \leq \lambda \quad(|z|<1)
$$

for some $\lambda \geq 0$ and $\mu>-1$. We set $\mathscr{U}(\lambda, 1)=\mathscr{U}(\lambda)$, and $\mathscr{U}(1)=\mathscr{U}$. In [10], the authors studied a subclass $\mathscr{P}(2 \lambda)$ of $\mathscr{U}(\lambda)$, consisting of functions $f$ for which

$$
\left|\left(\frac{z}{f(z)}\right)^{\prime \prime}\right| \leq 2 \lambda \quad(|z|<1) \text {. }
$$

We have the strict inclusion $\mathscr{P}(2) \subsetneq \mathscr{U} \subsetneq \mathscr{S}$, see $[1,7,13]$. Moreover, a close connection between the classes $\mathscr{P}(2 \lambda)$ and $\mathscr{U}(\lambda)$ is given by $\mathscr{P}(2 \lambda) \subset \mathscr{U}(\lambda)$, see $[9$, 10]. In $[8,14,15,16]$, the authors considered the problem of finding conditions on $\lambda$ and $\mu$ so that each function in $\mathscr{U}(\lambda, \mu)$ is starlike or in some subsets of $\mathscr{S}$. For example, Ponnusamy and Singh [15] have shown that

$$
\mathscr{U}(\lambda, \mu) \subseteq \mathscr{S}^{*} \text { if } \mu<0 \text { and } 0 \leq \lambda \leq \frac{1-\mu}{\sqrt{(1-\mu)^{2}+\mu^{2}}}=: \lambda^{*}(\mu)
$$

and in [8], Obradovic proved that the above inclusion continues to hold for $0<\mu \leq 1$ and with the same bound for $\lambda$. The sharpness part of these results may be obtained as a consequence of results from [21]. However, it is not known whether each function $f$ in $\mathscr{U}(1, \mu)$ (or more generally, $\mathscr{U}(\lambda, \mu)$ with $\left.\lambda^{*}(\mu)<\lambda \leq 1\right)$ is univalent in $\Delta$ for certain values of $\mu$ in the open interval $(0,1)$. On the other hand, according to a result due to Aksentiev [1] (see also Ozaki and Nunokawa [13] for a reformulated version as given by $\mathscr{U}$ ), we have the inclusion $\mathscr{U}(\lambda) \subset \mathscr{S}$ for $0 \leq \lambda \leq 1$. We see that the Koebe function $z /(1-z)^{2}$ belongs to $\mathscr{U}$ but does not belong to $\mathscr{S}^{*}(\alpha)$ for any $\alpha>0$. In fact, the bounded function $z+z^{2} / 2$ belongs to $\mathscr{U}$ but not in $\mathscr{S}^{*}(\alpha)$ for any $\alpha>0$. That is, $\mathscr{U} \not \subset \mathscr{S}^{*}(\alpha)$ for any $\alpha>0$. Thus, $\mathscr{U} \subsetneq \mathscr{S}$ and the inclusion is strict as functions in $\mathscr{S}$ are not necessarily in $\mathscr{U}$. Further work on these classes, including some interesting generalizations of these classes, may be found in $[9$, 12, 17].

A function $f \in \mathscr{S}^{*}(\alpha)$ is said to be in $\mathscr{T}^{*}(\alpha)$ if it can be expressed as

$$
f(z)=z-\sum_{k=2}^{\infty}\left|a_{k}\right| z^{k}
$$

Functions of this form are discussed in detail by Silverman [23] and others [24]. In this paper we shall be mainly concerned with functions $f \in \mathscr{A}$ of the form

$$
\left(\frac{z}{f(z)}\right)^{\mu}=1+\sum_{n=1}^{\infty} b_{n} z^{n}, \quad z \in \Delta
$$


where $(z / f(z))^{\mu}$ represents principal powers. The class of functions $f$ of this form for which $b_{n} \geq 0$ is especially interesting and deserves separate attention. We remark that if $f \in \mathscr{S}$ then $z / f(z)$ is nonvanishing and hence, $f \in \mathscr{S}$ may be expressed as

$$
f(z)=\frac{z}{g(z)}, \quad \text { where } g(z)=1+\sum_{n=1}^{\infty} c_{n} z^{n}, z \in \Delta .
$$

These two representations are convenient for our investigation. Finally, we introduce a subclass $\mathscr{S}_{p}(\alpha),-1 \leq \alpha \leq 1$, of starlike functions in the following way [19]:

$$
\mathscr{S}_{p}(\alpha)=\left\{f \in \mathscr{S}:\left|\frac{z f^{\prime}(z)}{f(z)}-1\right| \leq \operatorname{Re} \frac{z f^{\prime}(z)}{f(z)}-\alpha, z \in \Delta\right\} .
$$

Geometrically, $f \in \mathscr{S}_{p}(\alpha)$ if and only if the domain values of $z f^{\prime}(z) / f(z), z \in \Delta$, is the parabolic region $(\operatorname{Im} w)^{2} \leq(1-\alpha)[2 \operatorname{Re} w-(1+\alpha)$ ]. In [19], Rønning has shown that the class $\mathscr{S}_{p}(\alpha)$ must contain non-univalent functions if $\alpha<-1$, and $\mathscr{S}_{p}(\alpha) \subset \mathscr{S}^{*}$ if $-1 \leq \alpha \leq 1$. We set $\mathscr{S}_{p}(0)=\mathscr{S}_{p}$. The class of uniformly convex functions was introduced by Goodman in [4] (see also [5] where Goodman studied the class of uniformly starlike functions). Later Rønning [20] studied these classes along with the class $\mathscr{S}_{p}$. Moreover, from the work of Rønning [20], it follows easily that $f(z)=z+a_{n} z^{n}$ is in $\mathscr{S}_{p}(\alpha)$ if and only if $(2 n-1-\alpha)\left|a_{n}\right| \leq$ $1-\alpha$.

Let $\mathscr{F}$ and $\mathscr{G}$ be two subclasses of $\mathscr{A}$. If for every $f \in \mathscr{F}, r^{-1} f(r z) \in \mathscr{G}$ for $r \leq r_{0}$, and $r_{0}$ is the largest number for which this holds, then we say that $r_{0}$ is the $\mathscr{G}$ radius (or the radius of the property connected to $\mathscr{G}$ ) in $\mathscr{F}$. There are many results of this type in the theory of univalent functions. For example, the $\mathscr{S}_{p}$ radius in $\mathscr{S}^{*}$ was found by Rønning in [20] to be $1 / 3$. Also, $\mathscr{P}(2)$ radius in $\mathscr{U}$ has been obtained by Obradović and Ponnusamy in [11] and is given by $2 / 3$. At this place, it is appropriate to recall here the following result:

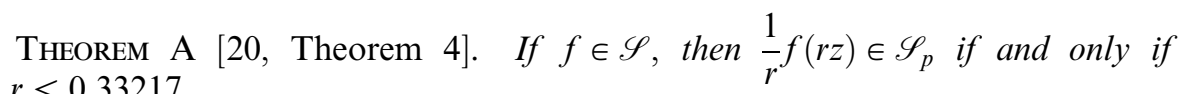
$0<r \leq 0.33217 \ldots$

\section{Lemmas}

For the proof of our results, we need the following result (see [3, Theorem 11 in p. 193 of Vol-2]) which reveals the importance of the area theorem in the theory of univalent functions.

Lemma 1. Let $\mu>0$ and $f \in \mathscr{S}$ be in the form (1.1). Then we have

$$
\sum_{n=1}^{\infty}(n-\mu)\left|b_{n}\right|^{2} \leq \mu \text {. }
$$


Next we recall well-known coefficient condition that is sufficient for functions to be in $\mathscr{U}(\lambda)$ or $\mathscr{P}(2 \lambda)$ or $\mathscr{S}^{*}(\alpha)$, respectively.

Lemma 2 [12]. Let $\phi(z)=1+\sum_{n=1}^{\infty} b_{n} z^{n}$ be a non-vanishing analytic function in $\Delta$ and $f(z)=z / \phi(z)$. Then if $\sum_{n=2}^{\infty}(n-1)\left|b_{n}\right| \leq \lambda$, we have

(a) $f \in \mathscr{U}(\lambda)$

(b) $f \in \mathscr{U}(\lambda) \cap \mathscr{S}^{*}$ for $0<\lambda \leq \frac{\sqrt{2-\left|b_{1}\right|^{2}}-\left|b_{1}\right|}{2}=\lambda_{*}(f)$;

(c) Further, if $\sum_{n=2}^{\infty} n(n-1)\left|b_{n}\right| \leq 2 \lambda$, then we have $f \in \mathscr{P}(2 \lambda)$.

In [18], it was shown that if $\phi(z)=1+\sum_{n=1}^{\infty} b_{n} z^{n}$ is a non-vanishing analytic function in $\Delta$ and $f(z)=z / \phi(z)$, then $f \in \mathscr{S}^{*}(\alpha), 0 \leq \alpha \leq 1$, whenever

$$
\sum_{k=2}^{\infty}(k-1+\alpha)\left|b_{k}\right| \leq \begin{cases}1-\alpha-(1-\alpha)\left|b_{1}\right| & \text { if } 0 \leq \alpha \leq 1 / 2 \\ 1-\alpha-\alpha\left|b_{1}\right| & \text { if } 1 / 2 \leq \alpha \leq 1\end{cases}
$$

\section{Coefficient conditions for functions in $\mathscr{S}_{p}(\alpha)$}

THEOREM 1. If a function $f$ of the form (1.1) with $b_{n} \geq 0$ and $\mu>0$ is in $\mathscr{S}_{p}(\alpha)$, we then have

$$
\sum_{n=1}^{\infty}(2 n-\mu(1-\alpha)) b_{n} \leq \mu(1-\alpha)
$$

Proof. Let $f \in \mathscr{S}_{p}(\alpha)$. Now, it is easy to see that

$$
z \frac{d}{d z}\left(\frac{z}{f(z)}\right)^{\mu}=\mu\left[\left(\frac{z}{f(z)}\right)^{\mu}-\left(\frac{z}{f(z)}\right)^{\mu+1} f^{\prime}(z)\right] .
$$

Using the identity (3.2), we have

$$
\begin{aligned}
\left|\frac{z f^{\prime}(z)}{f(z)}-1\right| & \leq \operatorname{Re}\left(\frac{z f^{\prime}(z)}{f(z)}\right)-\alpha \Leftrightarrow\left|\frac{-\frac{z}{\mu} \frac{d}{d z}\left(\frac{z}{f(z)}\right)^{\mu}}{\left(\frac{z}{f(z)}\right)^{\mu}}\right| \\
& \leq \operatorname{Re} \frac{\left(\frac{z}{f(z)}\right)^{\mu}-\frac{z}{\mu} \frac{d}{d z}\left(\frac{z}{f(z)}\right)^{\mu}}{\left(\frac{z}{f(z)}\right)^{\mu}}-\alpha .
\end{aligned}
$$

Since $f$ is in the form (1.1), the last inequality may be equivalently written as 


$$
\frac{1}{\mu}\left|\frac{-\sum_{n=1}^{\infty} n b_{n} z^{n}}{1+\sum_{n=1}^{\infty} b_{n} z^{n}}\right| \leq \operatorname{Re}\left(1-\frac{1}{\mu} \frac{\sum_{n=1}^{\infty} n b_{n} z^{n}}{1+\sum_{n=1}^{\infty} b_{n} z^{n}}\right)-\alpha .
$$

If $z \in \Delta$ is real and tends to $1^{-}$through reals, then from the last inequality we have

$$
\frac{1}{\mu}\left(\frac{\sum_{n=1}^{\infty} n b_{n}}{1+\sum_{n=1}^{\infty} b_{n}}\right) \leq 1-\alpha-\frac{1}{\mu}\left(\frac{\sum_{n=1}^{\infty} n b_{n}}{1+\sum_{n=1}^{\infty} b_{n}}\right),
$$

from which we obtain the desired inequality (3.1).

The case $\mu=1$ leads to

Corollary 1. Let $f \in \mathscr{S}_{p}(\alpha)$ be such that $z / f(z)=1+\sum_{n=1}^{\infty} b_{n} z^{n}$ with $b_{n} \geq 0$. Then we have

$$
\sum_{n=1}^{\infty}(2 n-1+\alpha) b_{n} \leq 1-\alpha
$$

THEOREM 2. Let $z / f(z)$ be a nonvanishing analytic function of the form (1.1) with $\mu>0$. Then the condition

$$
\sum_{n=1}^{\infty}(2 n+\mu(1-\alpha))\left|b_{n}\right| \leq \mu(1-\alpha)
$$

is sufficient for $f$ to be in the class $\mathscr{S}_{p}(\alpha)$.

Proof. As in the proof of Theorem 1, we notice that

$$
\left|\frac{z f^{\prime}(z)}{f(z)}-1\right| \leq \operatorname{Re}\left(\frac{z f^{\prime}(z)}{f(z)}\right)-\alpha
$$

is equivalent to

$$
\left|-\frac{\sum_{n=1}^{\infty} n b_{n} z^{n}}{1+\sum_{n=1}^{\infty} b_{n} z^{n}}\right| \leq \mu(1-\alpha)-\operatorname{Re}\left(\frac{\sum_{n=1}^{\infty} n b_{n} z^{n}}{1+\sum_{n=1}^{\infty} b_{n} z^{n}}\right) .
$$

Thus, to show that $f$ is in $\mathscr{S}_{p}(\alpha)$, it suffices to show that the quotient

$$
-\frac{\sum_{n=1}^{\infty} n b_{n} z^{n}}{1+\sum_{n=1}^{\infty} b_{n} z^{n}}
$$

lies in the parabolic region

$$
(\operatorname{Im} w)^{2} \leq \mu(1-\alpha)[\mu(1-\alpha)+2 \operatorname{Re} w] .
$$

Geometrically, this condition holds if we can show that 


$$
\left|\frac{\sum_{n=1}^{\infty} n b_{n} z^{n}}{1+\sum_{n=1}^{\infty} b_{n} z^{n}}\right| \leq \frac{\mu(1-\alpha)}{2}, \quad z \in \Delta .
$$

From the condition (3.3), we obtain that

$$
\sum_{n=1}^{\infty}(2 n+\mu(1-\alpha))\left|b_{n}\right||z|^{n} \leq \mu(1-\alpha)
$$

and so

$$
\sum_{n=1}^{\infty} n\left|b_{n}\right||z|^{n} \leq \frac{\mu(1-\alpha)}{2}\left(1-\sum_{n=1}^{\infty}\left|b_{n}\right||z|^{n}\right) .
$$

In view of this inequality, we deduce that

$$
\left|\frac{\sum_{n=1}^{\infty} n b_{n} z^{n}}{1+\sum_{n=1}^{\infty} b_{n} z^{n}}\right| \leq \frac{\mu(1-\alpha)}{2}\left(\frac{1-\sum_{n=1}^{\infty}\left|b_{n}\right||z|^{n}}{1-\sum_{n=1}^{\infty}\left|b_{n}\right||z|^{n}}\right)=\frac{\mu(1-\alpha)}{2}
$$

which is exactly the inequality (3.4) and therefore, $f \in \mathscr{S}_{p}(\alpha)$.

COROLlary 2. Let $z / f(z)$ be a nonvanishing analytic function in $\Delta$ of the form $z / f(z)=1+\sum_{n=1}^{\infty} b_{n} z^{n}$. Then the condition

$$
\sum_{n=1}^{\infty}(2 n+1-\alpha)\left|b_{n}\right| \leq 1-\alpha
$$

is sufficient for $f$ to be in the class $\mathscr{S}_{p}(\alpha)$.

The case $\alpha=0$ of Corollaries 1 and 2 has been obtained recently by Obradović and Ponnusamy [11].

\section{Radius problems}

THEOREM 3. If $f \in \mathscr{S}$ is given by (1.1) with $0<\mu<1$, then $\frac{1}{r} f(r z) \in \mathscr{S}_{p}(\alpha)$ for $0<r \leq r_{0}$, where $r_{0}$ is the root of the integral equation

$$
\frac{4 r^{2}\left(1+\mu(2-\alpha)\left(1-r^{2}\right)\right)}{\left(1-r^{2}\right)^{2}}+\frac{r^{2} \mu^{2}(3-\alpha)^{2}}{1-\mu} \int_{0}^{1} \frac{d t}{1-r^{2} t^{1 /(1-\mu)}}=\mu(1-\alpha)^{2} .
$$

Proof. Let $f \in \mathscr{S}$ be given by (1.1) with $0<\mu<1$. Then $z / f(z)$ is nonvanishing in $\Delta$ and for $0<r \leq 1$, we have

$$
\left(\frac{z}{\frac{1}{r} f(r z)}\right)^{\mu}=1+\left(b_{1} r\right) z+\left(b_{2} r^{2}\right) z^{2}+\cdots
$$


If

$$
S:=\sum_{n=1}^{\infty}(2 n+\mu(1-\alpha))\left|b_{n}\right| r^{n} \leq \mu(1-\alpha)
$$

for some $r$, then $\frac{1}{r} f(r z) \in \mathscr{S}_{p}(\alpha)$, by Theorem 2. Now, using the CauchySchwarz inequality and Lemma 1 , we see that

$$
\begin{aligned}
S & =\sum_{n=1}^{\infty} \sqrt{n-\mu}\left|b_{n}\right| \frac{2 n+\mu(1-\alpha)}{\sqrt{n-\mu}} r^{n} \\
& \leq\left(\sum_{n=1}^{\infty}(n-\mu)\left|b_{n}\right|^{2}\right)^{1 / 2}\left(\sum_{n=1}^{\infty} \frac{(2 n+\mu(1-\alpha))^{2}}{n-\mu} r^{2 n}\right)^{1 / 2} \\
& \leq \sqrt{\mu}\left(\sum_{n=1}^{\infty} \frac{(2 n+\mu(1-\alpha))^{2}}{n-\mu} r^{2 n}\right)^{1 / 2} \\
& =\sqrt{\mu}\left(\sum_{n=1}^{\infty} \frac{(2 n+\mu(1-\alpha))^{2}-\mu^{2}(3-\alpha)^{2}}{n-\mu} r^{2 n}+\mu^{2}(3-\alpha)^{2} \sum_{n=1}^{\infty} \frac{r^{2 n}}{n-\mu}\right)^{1 / 2} \\
& =\sqrt{\mu}\left(\sum_{n=1}^{\infty} 4\left(n+\mu(2-\alpha) r^{2 n}+\mu^{2}(3-\alpha)^{2} \sum_{n=1}^{\infty} \frac{r^{2 n}}{n-\mu}\right)^{1 / 2}\right. \\
& =\sqrt{\mu}\left(\frac{4 r^{2}\left(1+\mu(2-\alpha)\left(1-r^{2}\right)\right)}{\left(1-r^{2}\right)^{2}}+\frac{r^{2} \mu^{2}(3-\alpha)^{2}}{1-\mu} \int_{0}^{1} \frac{d t}{1-r^{2} t^{1 /(1-\mu)}}\right)^{1 / 2} .
\end{aligned}
$$

In particular, if the last expression is less than or equal to $\mu(1-\alpha)$, then $(4.2)$ holds which gives the condition (4.1).

In the case $\mu=1$, Theorem 3 takes the following form which needs a special attention as we see that the radius quantity depends on the second coefficient of the given function $f$.

THEOREM 4. If $f \in \mathscr{S}$ is of the form $z / f(z)=1+\sum_{n=1}^{\infty} b_{n} z^{n}$, then $\frac{1}{r} f(r z) \in$ $\mathscr{S}_{p}(\alpha)$ for $0<r \leq r_{0}$, where $r_{0}$, which depends on the second coefficient of $f$, is the root of the equation

$$
\frac{4 r^{4}\left(1+(3-\alpha)\left(1-r^{2}\right)\right)}{\left(1-r^{2}\right)^{2}}-(3-\alpha)^{2} r^{2} \ln \left(1-r^{2}\right)=\left(1-\alpha-(3-\alpha)(r / 2)\left|f^{\prime \prime}(0)\right|\right)^{2} \text {. }
$$

Proof. Note that, for $f \in \mathscr{S}$ satisfying $z / f(z)=1+\sum_{n=1}^{\infty} b_{n} z^{n}$, we have $b_{1}=-f^{\prime \prime}(0) / 2$. Proceeding exactly as in the proof of Theorem 3 (but with 
$\mu=1$ ) and by considering summation to run from 2 to $\infty$, we obtain the required conclusion. So we omit the details.

We remark that, the case $\alpha=0$ of Theorem 4 is due to Obradovic and Ponnusamy in [11].

Now we prove a generalized version of Lemma 2(a) which is useful to prove our next results.

Lemma 3. Let $0 \leq \alpha<1$ and $\phi(z)=1+\sum_{n=1}^{\infty} b_{n} z^{n}$ be a non-vanishing analytic function in $\Delta$ satisfying the coefficient condition

$$
\sum_{n=1}^{\infty}(n-1+\alpha)\left|b_{n}\right| \leq \lambda(1-\alpha)
$$

Then the function $f$ defined by the equation $(z / f(z))^{1-\alpha}=\phi(z)$ is in $\mathscr{U}(\lambda, 1-\alpha)$.

Proof. Let $f$ be given by $(z / f(z))^{1-\alpha}=\phi(z)$, where $\phi(z) \neq 0$ in $\Delta$, and we choose here the principal branch so that $(z / f(z))^{1-\alpha}$ at $z=0$ is 1 . Then the power series representation of $\phi$ and the coefficient condition (4.3), lead to

$$
\left|\left(\frac{z}{f(z)}\right)^{2-\alpha} f^{\prime}(z)-1\right|=\left|-\frac{1}{1-\alpha} \sum_{n=1}^{\infty}(n-1+\alpha) b_{n} z^{n}\right| \leq \lambda
$$

and therefore, by the definition of the class, $f$ is in $\mathscr{U}(\lambda, 1-\alpha)$.

The following result determines the $\mathscr{U}(\lambda, \mu)$ radius in $\mathscr{S}$.

THEOREM 5. Suppose that $f \in \mathscr{S}, 0 \leq \alpha<1, \lambda>0$ and

$$
r_{\alpha, \lambda}=\frac{\lambda \sqrt{2(1-\alpha)}}{\left[\sqrt{\left(\alpha+2 \lambda^{2}(1-\alpha)\right)^{2}+4 \lambda^{2}(1-\alpha)^{2}\left(1-\lambda^{2}\right)}+\left(\alpha+2 \lambda^{2}(1-\alpha)\right)^{1 / 2}\right.} \text {. }
$$

Then we have $\frac{1}{r} f(r z) \in \mathscr{U}(\lambda, 1-\alpha)$ for

$$
0<r \leq r_{\alpha, \lambda}
$$

In particular, $\frac{1}{r} f(r z) \in \mathscr{U}(1,1-\alpha)$ for $0<r \leq \sqrt{(1-\alpha) /(2-\alpha)}$. form

Proof. Let $f \in \mathscr{S}$. Then $z / f(z) \neq 0$ in $\Delta$. So, we may consider $f$ in the

$$
\left(\frac{z}{f(z)}\right)^{1-\alpha}=1+\sum_{n=1}^{\infty} b_{n} z^{n}
$$

Now, Lemma 1 gives 


$$
\sum_{n=1}^{\infty}(n-1+\alpha)\left|b_{n}\right|^{2} \leq 1-\alpha
$$

On the other side, for $0<r \leq 1$, we obtain from (4.5) that

$$
\left(\frac{z}{\frac{1}{r} f(r z)}\right)^{1-\alpha}=1+\sum_{n=1}^{\infty}\left(b_{n} r^{n}\right) z^{n} .
$$

According to Lemma 3, it suffices to verify the inequality

$$
\sum_{n=1}^{\infty}(n-1+\alpha)\left|b_{n} r^{n}\right| \leq \lambda(1-\alpha)
$$

for $0<r \leq r_{\alpha, \lambda}$. Now, as before, we have

$$
\begin{aligned}
\sum_{n=1}^{\infty}(n-1+\alpha)\left|b_{n} r^{n}\right| & \leq\left(\sum_{n=1}^{\infty}(n-1+\alpha)\left|b_{n}\right|^{2}\right)^{1 / 2}\left(\sum_{n=1}^{\infty}(n-1+\alpha) r^{2 n}\right)^{1 / 2} \\
& \leq \sqrt{1-\alpha}\left(\frac{r^{4}}{\left(1-r^{2}\right)^{2}}+\alpha \frac{r^{2}}{1-r^{2}}\right)^{1 / 2} \\
& =\sqrt{1-\alpha}\left(\frac{r}{1-r^{2}}\right)\left(\alpha+(1-\alpha) r^{2}\right)^{1 / 2} \\
& \leq \lambda(1-\alpha)
\end{aligned}
$$

if $\frac{r}{1-r^{2}} \sqrt{\alpha+(1-\alpha) r^{2}} \leq \lambda \sqrt{1-\alpha}$. Note that

$$
\frac{r}{1-r^{2}} \sqrt{\alpha+(1-\alpha) r^{2}} \leq \lambda \sqrt{1-\alpha}
$$

is equivalent to (4.4), and so we complete the proof.

\section{Conditions for functions to be in $\mathscr{U}(\lambda, \mu)$}

To present our next result, we consider the class of functions of Bazilevič type, see $[6,22]$. The result is simple and surprising as it identifies a subclass which lies in $\mathscr{U}(\lambda, \mu)$. This generalizes the result of Obradovic and Ponnusamy, see [11, Theorem 5].

THEOREM 6. Let $0<\mu \leq 1$. If $f \in \mathscr{S}$ is given by (1.1) with $b_{n} \geq 0$, and satisfies the condition that $\operatorname{Re}\left(f^{\prime}(z)\left(\frac{f(z)}{z}\right)^{\mu-1}\right)>0$. Then $f \in \mathscr{U}(1, \mu)$. 
Proof. Using the equation (3.2), we notice that

$$
\begin{aligned}
\operatorname{Re}\left(f^{\prime}(z)\left(\frac{f(z)}{z}\right)^{\mu-1}\right)>0 & \Leftrightarrow \operatorname{Re} \frac{\frac{z f^{\prime}(z)}{f(z)}}{\left(\frac{z}{f(z)}\right)^{\mu}}>0 \\
& \Leftrightarrow \operatorname{Re} \frac{\left(\frac{z}{f(z)}\right)^{\mu}-\frac{z}{\mu} \frac{d}{d z}\left(\frac{z}{f(z)}\right)^{\mu}}{\left(\frac{z}{f(z)}\right)^{2 \mu}}>0 \\
& \Leftrightarrow \operatorname{Re} \frac{1+\sum_{n=1}^{\infty}(1-n / \mu) b_{n} z^{n}}{\left(1+\sum_{n=1}^{\infty} b_{n} z^{n}\right)^{2}}>0 .
\end{aligned}
$$

Since $b_{n} \geq 0$, allow $z \rightarrow 1^{-}$along the real axis, we get

$$
\operatorname{Re} \frac{1-\sum_{n=1}^{\infty}(n / \mu-1) b_{n}}{\left(1+\sum_{n=1}^{\infty} b_{n}\right)^{2}} \geq 0
$$

which gives that

$$
\sum_{n=1}^{\infty}(n-\mu) b_{n} \leq \mu
$$

and so by Lemma 3 , we have $f \in \mathscr{U}(1, \mu)$.

THeOrem 7. Let $0<\mu \leq 1$. A function $f$ of the form (1.1) with $b_{n} \geq 0$ and $z / f(z) \neq 0$, is in $\mathscr{U}(1, \mu)$ if and only if

$$
\sum_{n=1}^{\infty}(n-\mu) b_{n} \leq \mu
$$

Proof. In view of Lemma 3, it suffices to prove the necessary part. To do this, we let $f \in \mathscr{U}(1, \mu)$ and $f$ is of the form (1.1). Then using (3.2), we get

$$
\left|\left(\frac{z}{f(z)}\right)^{\mu+1} f^{\prime}(z)-1\right|=\left|\left(\frac{z}{f(z)}\right)^{\mu}-\frac{z}{\mu} \frac{d}{d z}\left(\frac{z}{f(z)}\right)^{\mu}-1\right|=\frac{1}{\mu}\left|\sum_{n=1}^{\infty}(n-\mu) b_{n} z^{n}\right| \leq 1 .
$$

Because $b_{n} \geq 0$, choosing values of $z$ on the real axis and then letting $z \rightarrow 1^{-}$ through real values, we obtain the coefficient condition (5.1).

The following result gives a sufficient condition for starlike functions of order $\alpha$ to be in the class $\mathscr{U}(\lambda, \mu)$. 
THEOREM 8. If $f \in \mathscr{S}^{*}(\alpha)$ is of the form (1.1) with $b_{n} \geq 0$ and $\mu>0$, then

$$
\sum_{n=1}^{\infty}(n-\mu(1-\alpha)) b_{n} \leq \mu(1-\alpha) .
$$

In particular, $f \in \mathscr{U}(1-\alpha, \mu)$.

Proof. It is easy to see that

$$
f \in \mathscr{S}^{*}(\alpha) \Leftrightarrow \operatorname{Re}\left(\frac{z f^{\prime}(z)}{f(z)}\right) \geq \alpha \Leftrightarrow\left|\frac{\frac{z f^{\prime}(z)}{f(z)}-1}{\frac{z f^{\prime}(z)}{f(z)}+1-2 \alpha}\right| \leq 1 .
$$

Now, using this relation and the identity (3.2), we have the following

$$
\begin{aligned}
& \left|\frac{\frac{z f^{\prime}(z)}{f(z)}-1}{\frac{z f^{\prime}(z)}{f(z)}+1-2 \alpha}\right|=\left|\frac{-z \frac{d}{d z}\left(\frac{z}{f(z)}\right)^{\mu}}{2 \mu(1-\alpha)\left(\frac{z}{f(z)}\right)^{\mu}-z \frac{d}{d z}\left(\frac{z}{f(z)}\right)^{\mu}}\right| \\
& =\left|\frac{-\sum_{n=1}^{\infty} n b_{n} z^{n}}{2 \mu(1-\alpha)\left(1+\sum_{n=1}^{\infty} b_{n} z^{n}\right)-\sum_{n=1}^{\infty} n b_{n} z^{n}}\right| \leq 1 .
\end{aligned}
$$

Since $b_{n} \geq 0$, if $z \rightarrow 1^{-}$along the real axis, we see from the last inequality that

$$
\frac{\sum_{n=1}^{\infty} n b_{n}}{2 \mu(1-\alpha)-\sum_{n=1}^{\infty}(n-2 \mu(1-\alpha)) b_{n}} \leq 1 .
$$

This gives the desired inequality (5.2).

Finally, since $n-\mu \leq n-\mu(1-\alpha)$, we have

$$
\sum_{n=1}^{\infty}(n-\mu) b_{n} \leq \sum_{n=1}^{\infty}(n-\mu(1-\alpha)) b_{n} \leq \mu(1-\alpha) .
$$

From Lemma 3, we conclude that $f \in \mathscr{U}(1-\alpha, \mu)$.

As a consequence of Theorem 8 , we next see that $\mathscr{T}^{*}(\alpha) \subset \mathscr{U}(1-\alpha)$.

Corollary 3. If $f(z)=z-\sum_{n=2}^{\infty}\left|a_{n}\right| z^{n}$ is in $\mathscr{S}^{*}(\alpha)$, then $f \in \mathscr{U}(1-\alpha)$.

Proof. Let $f \in \mathscr{S}^{*}(\alpha)$ be of the form $f(z)=z-\sum_{n=2}^{\infty}\left|a_{n}\right| z^{n}$. Then $z / f(z)$ is nonvanishing in the unit disk and so it can be expressed as 


$$
\frac{z}{f(z)}=\frac{1}{1-\left|a_{2}\right| z-\left|a_{3}\right| z^{2}-\cdots}=1+b_{1} z+b_{2} z^{2}+\cdots,
$$

where $b_{n} \geq 0$ for all $n \in \mathbf{N}$. Then by Theorem $8, f \in \mathscr{U}(1-\alpha)$.

From [12], we collect the following result.

Lemma 4. Let $0 \leq \lambda, \gamma \leq 1$ and $f \in \mathscr{U}(\lambda)$. Define

$$
\lambda_{\gamma}^{*}=\frac{-\left|f^{\prime \prime}(0)\right| \cos (\pi \gamma / 4)+\sin (\pi \gamma / 4) \sqrt{16 \cos ^{2}(\pi \gamma / 4)-\left|f^{\prime \prime}(0)\right|^{2}}}{2 \cos (\pi \gamma / 4)}
$$

and let $\lambda_{\gamma}^{\mathscr{R}}$ be given by the inequality

$$
\sin (\pi \gamma / 2) \sqrt{4-\lambda^{2}} \geq\left(\left|f^{\prime \prime}(0)\right|+\lambda\right) \sqrt{4-\left(\left|f^{\prime \prime}(0)\right|+\lambda\right)^{2}}+\lambda \cos (\pi \gamma / 2) .
$$

Then

(i) $f \in \mathscr{U}(\lambda) \Rightarrow f \in \mathscr{S}_{\gamma}$ for $0<\lambda \leq \lambda_{\gamma}^{*} / 2$, where

(ii) $f \in \mathscr{U}(\lambda) \Rightarrow f \in \mathscr{R}_{\gamma}$ for $0<\lambda \leq \lambda_{\gamma}^{\mathscr{R}} / 2$,

$$
\begin{aligned}
& \mathscr{R}_{\gamma}:=\left\{f \in \mathscr{A}:\left|\arg f^{\prime}(z)\right| \leq \frac{\pi \gamma}{2}\right\} \text { and } \\
& \mathscr{S}_{\gamma}:=\left\{f \in \mathscr{A}:\left|\arg \left(z f^{\prime}(z) / f(z)\right)\right| \leq \frac{\pi \gamma}{2}\right\} .
\end{aligned}
$$

Using the containment results of Lemma 4 and Corollary 3, one can derive a number of interesting results. For instance, we obtain the following:

Corollary 4. If $0 \leq \gamma \leq 1$ and $f(z)=z-\sum_{n=3}^{\infty}\left|a_{n}\right| z^{n} \in \mathscr{S}^{*}\left(1-\sin \frac{\pi \gamma}{6}\right)$, then $f \in \mathscr{R}_{\gamma}$. In particular, if $f^{\prime \prime}(0)=0$, then $f \in \mathscr{S}^{*}(1 / 2)$ implies that $\operatorname{Re} f^{\prime}(z) \geq 0$.

COROLlary 5. If $0 \leq \gamma \leq 1$ and $f(z)=z-\sum_{n=3}^{\infty}\left|a_{n}\right| z^{n} \in \mathscr{S}^{*}\left(1-\sin \frac{\pi \gamma}{4}\right)$, then $f \in \mathscr{S}_{\gamma}$. In particular, if $f^{\prime \prime}(0)=0$, then $f \in \mathscr{S}^{*}(1 / 2)$ implies that $\left|\arg \left(z f^{\prime}(z) / f(z)\right)\right| \leq \pi / 3$.

\section{Conclusion}

To present a meromorphic analog of the class $\mathscr{U}(\lambda)$, we recall, for example, the following result.

Lemma 5 [17, Theorem 1.2]. If $f \in \mathscr{U}(\lambda)$ and $a=\left|f^{\prime \prime}(0)\right| / 2 \leq 1$, then $f \in \mathscr{S}^{*}(\delta)$ whenever $0 \leq \lambda \leq \lambda(\delta)$, where 


$$
\lambda(\delta)= \begin{cases}\frac{\sqrt{(1-2 \delta)\left(2-a^{2}-2 \delta\right)}-a(1-2 \delta)}{2(1-\delta)} & \text { if } 0 \leq \delta<\frac{1+a}{3+a}, \\ \frac{1-\delta(1+a)}{1+\delta} & \text { if } \frac{1+a}{3+a} \leq \delta<\frac{1}{1+a} .\end{cases}
$$

In particular,

$$
f \in \mathscr{U}(\lambda), f^{\prime \prime}(0)=0 \Rightarrow f \in \mathscr{S}^{*} \text { whenever } 0 \leq \lambda \leq 1 / \sqrt{2} .
$$

After the paper was submitted to the journal, Fournier and Ponnusamy [2] settled the question of sharpness of the bound for $\lambda$ for which $\mathscr{U}(\lambda) \subset \mathscr{S}^{*}$. As a motivation for our next result, we consider the class, denoted by $\Sigma$, of all functions of the form

$$
F(\zeta)=\zeta+\sum_{n=0}^{\infty} c_{n} \zeta^{-n}
$$

that are analytic and univalent for $|\zeta|>1$. Thus

$$
F \in \Sigma \Leftrightarrow f \in \mathscr{S}, \quad f(z)=\frac{1}{F(1 / z)}=\frac{z}{1+\sum_{n=1}^{\infty} c_{n-1} z^{n}} .
$$

Also, we note that

$$
f^{\prime}(z)\left(\frac{z}{f(z)}\right)^{2}=F^{\prime}(1 / z) \text { and } \frac{z f^{\prime}(z)}{f(z)}=\frac{(1 / z) F^{\prime}(1 / z)}{F(1 / z)} .
$$

Consequently, for $0 \leq \lambda \leq 1, f \in \mathscr{U}(\lambda)$ if and only if $\left|F^{\prime}(\zeta)-1\right| \leq \lambda$ for $|\zeta|>1$. Similarly, for $0 \leq \alpha \leq 1, f \in \mathscr{S}^{*}(\alpha)$ if and only if

$$
\operatorname{Re}\left(\frac{\zeta F^{\prime}(\zeta)}{F(\zeta)}\right) \geq \alpha \text { for }|\zeta|>1
$$

The class of all such functions satisfying the later condition is denoted by $\Sigma^{*}(\alpha)$. Thus, Lemma 5 takes the following form:

THEOREM 9. Let $F(\zeta)=\zeta+\sum_{n=0}^{\infty} c_{n} \zeta^{-n}$ be analytic and univalent for $|\zeta|>1$. If $F$ satisfies the condition

$$
\left|F^{\prime}(\zeta)-1\right| \leq \lambda \quad \text { for }|\zeta|>1
$$

and $a=\left|-c_{0}\right| \leq 1$, then $F \in \Sigma^{*}(\delta)$ whenever $0<\lambda \leq \lambda(\delta)$, where $\lambda(\delta)$ is given by (6.1). In particular, for $c_{0}=0, F \in \Sigma^{*}(\delta)$ whenever $0<\lambda \leq 1 / \sqrt{2}$.

In view of the above observations, we can state a number of results for various subclasses of the class of meromorphic univalent functions.

\section{REFERENCES}

[1] L. A. Aksentiev, Sufficient conditions for univalence of regular functions (in Russian), Izv. Vysš. Učebn. Zaved. Matematika 3 (4) (1958), 3-7. 
[2] R. Fournier and S. Ponnusamy, A class of locally univalent functions defined by a differential inequality, to appear in Complex Variables Theory and Appl.

[ 3 ] A. W. Goodman, Univalent functions 1 and 2, Mariner, Tampa, Florida, 1983.

[4] A. W. Goodman, On uniformly convex functions, Ann. Polon. Math. 56 (1991), 87-92.

[ 5] A. W. Goodman, On uniformly starlike functions, J. Math. Anal. Appl. 155 (1991), 364-370.

[6] Jian-Lin LI, Notes on the starlikeness of an integral transform, Tamkang J. Math. 32 (2001), $151-154$.

[7] M. Nunokawa, M. Obradović and S. Owa, One criterion for univalency, Proc. Amer. Math. Soc. 106 (1989), 1035-1037.

[ 8 ] M. Obradović, A class of univalent functions, Hokkaido Math. J. 27 (1998), 329-335.

[9] M. Obradović and S. Ponnusamy, New criteria and distortion theorems for univalent functions, Complex Variables Theory Appl. 44 (2001), 173-191.

[10] M. Obradović and S. Ponnusamy, Radius properties for subclasses of univalent functions, Analysis (Munich) 25 (2005), 183-188.

[11] M. Obradović AND S. Ponnusamy, On certain subclasses of univalent functions and radius properties, preprint.

[12] M. Obradović, S. Ponnusamy, V. Singh and P. Vasundhra, Univalency, starlikeness and convexity applied to certain classes of rational functions, Analysis (Munich) 22 (2002), 225-242.

[13] S. Ozaki and M. Nunokawa, The Schwarzian derivative and univalent functions, Proc. Amer. Math. Soc. 33 (1972), 392-394.

[14] S. Ponnusamy, Pólya Schoenberg conjecture by Carathéodory functions, J. London Math. Soc. (2) 51 (1995), 93-104.

[15] S. Ponnusamy AND V. Singh, Convolution properties of some classes analytic functions, Zapiski Nauchnych Seminarov POMI 226 (1996), 138-154.

[16] S. Ponnusamy and V. Singh, Criteria for strongly starlike functions, Complex Variables Theory Appl. 34 (1997), 267-291.

[17] S. Ponnusamy and P. Vasundhra, Criteria for univalence, starlikeness and convexity, Ann. Polon. Math. 85 (2005), 121-133.

[18] M. O. Reade, H. Silverman and P. G. Todorov, On the starlikeness and convexity of a class of analytic functions, Rend. Circ. Mat. Palermo (2) 33 (1984), 265-272.

[19] F. RønNing, On starlike functions associated with parabolic regions, Ann. Univ. Mariae Curie-Skłodowska Sect. A. 45 (1991), 117-122.

[20] F. RønNING, Uuniformly convex functions and a corresponding class of starlike functions, Proc. Amer. Math. Soc. 118 (1993), 189-196.

[21] F. RønNing, St. Ruscheweyh and N. SAmaris, Sharp starlikeness conditions for analytic functions with bounded derivative, J. Austral. Math. Soc. Ser. A. 69 (2000), 303-315.

[22] T. SheIl-SMall, Some remarks on Bazilevič functions, J. d'Analyse Math. 43 (1983/84), 1-11.

[23] H. Silverman, Univalent functions with negative coefficicents, Proc. Amer. Math. Soc. 51 (1975), 109-116.

[24] H. Silverman, E. M. Silvia and D. N. Telage, Locally univalent functions and coefficient distortions, Pacific J. Math. 77 (1978), 533-539.

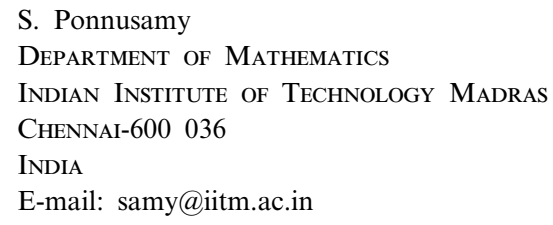


S. K. Sahoo

DEPARTMENT OF MATHEMATICS

Indian Institute of TeChNOLOGY MadRas

ChenNai-600 036

INDIA

E-mail: swadesh_iitm@math.net 